\title{
Sedum sarmentosum Bunge extract induces apoptosis and inhibits proliferation in pancreatic cancer cells via the hedgehog signaling pathway
}

\author{
YONGHENG BAI ${ }^{1,2}$, BICHENG CHEN ${ }^{2}$, WEILONG HONG $^{2}$, YONG LIANG ${ }^{2}$, \\ MENGTAO ZHOU $^{3}$ and LAN ZHOU ${ }^{1}$
}

\begin{abstract}
${ }^{1}$ Key Laboratory of Clinical Laboratory Diagnostics, Ministry of Education, College of Clinical Laboratory Diagnostics, Chongqing Medical University, Chongqing 400016; ${ }^{2}$ Key Laboratory of Surgery and ${ }^{3}$ Department of Hepatobiliary Surgery, The First Affiliated Hospital, Wenzhou Medical University, Wenzhou, Zhejiang 325000, P.R. China
\end{abstract}

Received December 10, 2015; Accepted January 12, 2016

DOI: 10.3892/or.2016.4679

\begin{abstract}
Sedum sarmentosum Bunge, a traditional Chinese herbal medicine, has a wide range of clinical applications including antibiosis, anti-inflammation and anti-oxidation. In the present study, we identified that its extract (SSBE) exerts pancreatic anticancer activity in vitro and in vivo. In the cultured pancreatic cancer PANC-1 cell line, SSBE inhibited cell growth in a concentration-dependent manner, and it was accompanied by the downregulated expression of proliferating cell nuclear antigen (PCNA). In addition, SSBE treatment also increased cellular apoptosis in a mitochondrial-dependent manner. Moreover, SSBE induced p53 expression, reduced c-Myc expression, and inhibited epithelial-mesenchymal transition (EMT). The antiproliferative activity of SSBE in the pancreatic cancer cells was found to be closely related to cell cycle arrest at the G2/M phase by upregulating p21(Waf1/ CIP1) expression. Further study showed that this inhibitory effect of SSBE was through downregulation of the activity of the proliferation-related Hedgehog signaling pathway. Exogenous recombinant protein Shh was used to activate Hedgehog signaling, thereby resulting in the abolishment of the SSBE-mediated inhibition of pancreatic cancer cell growth. In animal xenograft models of pancreatic cancer, activated Hedgehog signaling was also observed compared with the vehicle controls, but was reduced by SSBE administration. As a result, SSBE suppressed the growth of pancreatic tumors. Thus, these findings demonstrate that SSBE has therapeutic potential for pancreatic cancer, and this anticancer effect in
\end{abstract}

Correspondence to: Dr Lan Zhou, Key Laboratory of Clinical Laboratory Diagnostics, Ministry of Education, College of Clinical Laboratory Diagnostics, Chongqing Medical University, Chongqing 400016, P.R. China

E-mail: greatsailor@163.com

Key words: Sedum sarmentosum Bunge, pancreatic cancer, cell cycle, proliferation, Hedgehog signaling pancreatic cancer cells is associated with inhibition of the Hedgehog signaling pathway.

\section{Introduction}

Pancreatic cancer is the most devastating of human digestive tract malignant tumors. The high lethality of pancreatic cancer is primarily due to several reasons, including inapparent early symptoms and the absence of effective screening tests, thereby leading to a late presentation of disease and the poor responsiveness of the tumor to existing therapies (1). To date, surgical resection is the only potentially curative therapeutic option. However, the majority of patients present with metastatic disease, rendering their malignancy inoperable (2). Conventional chemotherapy is regarded as a widely applied strategy for the non-surgical treatment of pancreatic cancer. However, it is still rarely curative for patients with advanced stages of the disease, particularly in cases of metastatic pancreatic cancer (3). Therefore, novel and effective regimens against pancreatic cancer are urgently needed.

Chinese herbal medicine has been proven to be a valuable resource of potential anticancer agents with minimal toxicity $(4,5)$. Sedum sarmentosum Bunge (SSB) is a perennial herb widely distributed on the mountain slopes in Asian countries and is traditionally used for the treatment of various inflammatory diseases $(6,7)$. The extract of SSB (SSBE) contains multiple active chemical components, including quercetin and isorhamnetin (8-10). Quercetin is a plant-derived flavonoid that has potential antiviral $(11)$, anticancer $(12,13)$ and anti-inflammatory properties (14). Isorhamnetin is an $O$-methylated flavonol, a type of chemical compound that exhibits biological activity such as anti-oxidant, anticancer and improvement of cardiovascular function (15). Thus, these findings support the concept that SSBE may have potent anticancer activity in various tissues, including the pancreas.

However, a study on the role of the pancreatic anticancer effects of SSBE has not been performed. Therefore, in the present study, for the first time, we investigated the effects of SSBE on pancreatic cancer cells (PCCs) in vitro by evaluating cellular proliferation and apoptosis, cell cycle and 
epithelial-mesenchymal transition (EMT), and in vivo by observing the tumor development of animal xenograft models of pancreatic cancer. Furthermore, we also examined the expression levels of cytokines involved in cell cycle regulation in cultured PCCs, evaluating the involvement of the Hedgehog signaling pathway.

\section{Materials and methods}

Cell culture. The human pancreatic cancer PANC-1 cell line was obtained from the Cell Bank of the Chinese Academy of Sciences (Shanghai, China). PANC-1 cells were maintained in Dulbecco's modified Eagle's medium (DMEM) supplemented with $5 \%$ fetal bovine serum (FBS), $100 \mu \mathrm{g} / \mathrm{ml}$ streptomycin and $100 \mathrm{U} / \mathrm{ml}$ penicillin (all from Invitrogen, Carlsbad, CA, USA). The PANC-1 cells were seeded on 6-well culture plates to $50-70 \%$ confluency in complete medium containing $5 \%$ FBS for $24 \mathrm{~h}$, and then changed to serum-free medium for $24 \mathrm{~h}$ before treatment with SSBE (Lot no. 20101017; Xuancheng Baicao Plant Industry and Trade Co., Ltd., Anhui, China). The extraction protocol of SSB was carried out according to a previous study (16).

CCK- 8 assay. Viability of the PANC-1 cells treated with SSBE were measured using the CCK-8 cell proliferation and cytotoxicity assay kit (Dojindo, Shanghai, China) according to the manufacturer's instructions. At the indicated time points, $10 \mu \mathrm{l}$ of this reagent at different concentrations was added to each well containing $100 \mu \mathrm{l}$ of the cell suspension $\left(5 \times 10^{3}\right.$ cells) and incubated for an additional $1 \mathrm{~h}$. The absorbance at $450 \mathrm{~nm}$ was monitored, and the percent viability of the cells was calculated by comparison to that of the untreated control cells. All the experiments were repeated at least three times.

Flow cytometric analysis. The PANC-1 cells treated with SSBE were seeded for $24 \mathrm{~h}$, and then were collected by centrifugation. For apoptosis analysis, resuspended cells were incubated with Annexin V-FITC and propidium iodide (PI) at room temperature for $5 \mathrm{~min}$ in the dark. Analysis of Annexin V-FITC binding by flow cytometery (Ex, $488 \mathrm{~nm}$; Em, $530 \mathrm{~nm}$; BD FACSVerse $^{\mathrm{TM}}$ ) (BD Biosciences, Frankin Lakes, NJ, USA) was accomplished using FITC signal detector and PI staining by the phycoerythrin emission signal detector. For cell cycle analysis, resuspended cells were fixed in $70 \%$ ethanol at $4^{\circ} \mathrm{C}$ overnight and permeated in $0.1 \%$ Triton $\mathrm{X}-100$ at $4^{\circ} \mathrm{C}$ for $30 \mathrm{~min}$. After incubating with PI at $4^{\circ} \mathrm{C}$ for $30 \mathrm{~min}, \mathrm{PCCs}$ were analyzed using flow cytometry and the proportion of nuclei in each phase of the cell cycle was determined using ModFit LT 3.2 software (Verity Software House, Topsham, ME, USA).

Immunofluorescence staining. The PANC-1 cells were cultured with SSBE on 6-well plates containing glass slides, and were washed in phosphate-buffered saline (PBS) and fixed in $4 \%$ paraformaldehyde (Sigma-Aldrich, St. Louis, $\mathrm{MO}$, USA) at $4^{\circ} \mathrm{C}$ for $30 \mathrm{~min}$. After permeabilization in $0.1 \%$ Triton $\mathrm{X}-100$ for $10 \mathrm{~min}$, specimens were washed in PBS, and then the substrate was blocked with $10 \%$ FBS to eliminate the nonspecific fluorescence. Immunofluorescence staining was performed according to a previous study (17) using antibodies against proliferating cell nuclear antigen
(PCNA; 1:200; Santa Cruz Biotechnology, Santa Cruz, CA, USA), c-Myc (1:100; Biogot Technology, Shanghai, China), $\alpha$-SMA (1:200), p21 (1:200), Ptch1 (1:200) and Smo (1:200) (all from Santa Cruz Biotechnology) as the primary antibodies at $4^{\circ} \mathrm{C}$ overnight. After washing in PBS three times, the cell preparations were incubated with DyLight 488 (green)/594 (red)-labeled secondary antibodies (Sigma-Aldrich) for $1 \mathrm{~h}$ at room temperature. After being washed in PBS, the cell preparations were dropped in appropriate acacia and covered with a slide. Immunocytochemical studies were semi-quantitatively or quantitatively assessed by two independent investigators in a blinded manner.

Western blot analysis. Western blot analyses were examined according to a previous study (18). Whole proteins from PANC-1 cells were collected and the protein concentrations were determined using a bicinchoninic acid protein assay kit (Beyotime Biotechnology). Whole proteins $(20 \mu \mathrm{g})$ from each sample were separated by SDS-PAGE and transferred to a polyvinylidene difluoride membrane (Solarbio, Beijing, China). After treatment with $5 \%$ skim milk at $4^{\circ} \mathrm{C}$ overnight, the membranes were incubated with various antibodies for $1 \mathrm{~h}$, and then incubated with the appropriate horseradish peroxidase-conjugated secondary antibody (Beyotime). Bound antibodies were visualized using chemiluminescence detection on autoradiographic film. Primary antibodies were as follows: polyclonal anti-Bcl-2 (1:400), Bax (1:400), Bcl-2 (1:400), caspase-3 (1:400), caspase-8 (1:400) (all from Beyotime), p53 (1:500; Biogot), p21 (1:200; Biotechnology) and c-Myc (1:500; Biogot). Quantification was performed by measuring the intensity of signals using Image-Pro Plus (version 6.0), and normalized to that for GAPDH (1:10,000; Cell Signaling Technology, Danvers, MA, USA).

Nude mouse tumorigenicity assay. Male nude mice (BALB/c) weighing 18-22 $\mathrm{g}$ and 6-8 weeks old were purchased from the Experimental Animal Centre of Wenzhou Medical University (Wenzhou, China). Mice were housed in a temperature-, humidity- and light-controlled environment, and fed a standard mouse chow and water. Mice were fasted on the day prior to the experiments being conducted. Before the experiments, all mice were anesthetized by an intraperitoneal injection with $0.2 \%$ pentobarbital natrium. The left neck of the experimental mice $(n=12)$ were subcutaneously injected with $5 \times 10^{6}$ PANC-1 cells in $10 \mu \mathrm{l}$ of PBS and then received daily intragastric administration of SSBE (10 and $100 \mathrm{mg} / \mathrm{kg} \cdot$ day) for 30 days. Model mice $(n=12)$ received an injection of $5 \times 10^{6}$ PANC-1 cells and daily gastric tube of solvent (PBS), and the healthy group $(n=6)$ was treated with PBS only. Tumors were monitored daily until they became cumbersome or necrotic. Tumor volume (V) was measured every other day based on the formula: $\mathrm{V}=$ length ${ }^{2} \mathrm{x}$ width, where length was always the longest dimension. After the experiments, all mice were euthanized by immersion in an ice-water bath and then burned. The animal study protocols including the method involving animal euthanasia were approved by the Institutional Animal Care and Use Committee of Wenzhou Medical University, China. The methods were also performed according to the guidelines approved by the Institutional Review Board of Wenzhou Key Laboratory of Surgery, China. 
Table I. Two-step real-time RT-PCR primers for analysis.

\begin{tabular}{lllc}
\hline Gene & \multicolumn{1}{c}{ Forward sequence $\left(5^{\prime} \rightarrow 3^{\prime}\right)$} & \multicolumn{1}{c}{ Reverse sequence $\left(5^{\prime} \rightarrow 3^{\prime}\right)$} & Product size $(\mathrm{bp})$ \\
\hline Smo & AGTTACATCGCAGCCTTC & CACACTACTCCAGCCATC & 299 \\
Gli1 & TGCTGACACTCTGGGATA & CAGGGCCATAGTTGGTT & 132 \\
Ptch1 & TGGTCACACGAACAATGG & TGAACTGGGCAGCTATGAAGTC & 202 \\
Survivin & CACCGCATCTCTACATTCAAG & CGGACGAATGCTTTTTATGT & 268 \\
TP53 & TCACCATCATCACACTGGAAGACTC & TTGGGCAGTGCTCGCTTAGT & 175 \\
Bax & TTTCTGACGGCAACTTCAACTG & CGGAGGAAGTCCAATGTCCAG & 136 \\
Bad & CTCCGGCAAGCATCATCG & CCCATCCCTTCGTCGTCCT & 162 \\
Bcl-2 & CAACACAGACCCACCCAGA & TGGCTTCATACCACAGGTTC & 134 \\
c-Myc & CCTCCACTCGGAAGGACTATC & TTCGCCTCTTGACATTCTCC & 135 \\
CCND1 & CCTGTCCTACTACCGCCTCA & TCCTCCTCTTCCTCCTCCTC & 165 \\
E-cadherin & TGGACCGAGAGAGTTTCC & AATATGGTGTATACAGCCTC & 250 \\
$\alpha-$ SMA & CGTGGCTACTCCTTCGTG & TGATGACCTGCCCGTCT & 160 \\
GAPDH & TCCCATCACCATCTTCCAGG & GATGACCCTTTTGGCTCCC & 145 \\
\hline
\end{tabular}

Histopathological examination. The tumor specimens were fixed in formalin and embedded in paraffin and then cut into $4-\mu \mathrm{m}$ sections and stained with hematoxylin and eosin (H\&E; Yuanye Biotechnology, Shanghai, China). Slides were examined and images were captured using a DM4000 B LED microscope system and a DFC420C 5M digital microscope camera (both from Leica Microsystems, Wetzlar, Germany).

Quantitative reverse transcriptase-PCR ( $q R T-P C R)$. Total RNA was extracted from the PANC-1 cells or tumor tissues using TRIzol reagent (Invitrogen), and reverse-transcribed to cDNA templates using a ReverTra Ace qPCR RT kit (Toyobo, Osaka, Japan). qRT-PCR was performed using SYBR-Green Real-Time PCR Master Mix Plus (Toyobo). Quality was analyzed on agarose gels, and quantities were measured using a Varioskan Flash (Thermo Fisher Scientific, Inc., Waltham, MA, USA). Sequence-specific primers for TP53, c-Myc, E-cadherin, $\alpha$-SMA, Bcl-2, Bax, Bad, survivin, CCND1, Smo, Ptch1 and Gli1 (Table I) were synthesized by Invitrogen and GAPDH was used as an endogenous reference gene. Samples were analyzed in triplicate, and the melting curve was examined to verify that a single product was amplified. For quantitative analysis, all samples were analyzed using the $\Delta \Delta \mathrm{CT}$ value method.

Statistical analysis. Data are presented as the mean \pm standard error of the mean. All statistical analyses were performed using a Statistical Package for Social Sciences (version 16.0; SPSS, Inc., Chicago, IL, USA). A two-sided Student's t-test was used to analyze differences between the two groups. A one-way analysis of variance was used when $>2$ groups were present. A P-value of $<0.05$ was considered to indicate a statistically significant result.

\section{Results}

Effect of SSBE on the proliferation and apoptosis of PCCs. To investigate whether SSBE exerts a protective effect on pancreatic cancer, we first evaluated cell proliferation in the SSBE-treated PCCs. As shown in Fig. 1A, evidence from phase contrast microscopy showed that the injury of PANC-1 cells induced by SSBE was significantly aggravated, and cell number was decreased. CCK-8 assay revealed that this inhibitory effect of SSBE on cell proliferation was concentration-dependent, but not time-dependent (Fig. 1B). In addition, immunofluorescence staining indicated that SSBE treatment significantly downregulated the expression of PCNA (Fig. 1C). Thus, these findings suggested that SSBE has a marked inhibitory effect on the proliferation of PCCs.

Secondly, we examined the effect of SSBE on cellular apoptosis. In the SSBE-treated PANC-1 cells, marked cellular apoptosis was observed. As shown in Fig. 1D, the number of apoptotic cells (including early and late apoptotic and necrotic cells) in the controls was $8.77 \%$, but reached $14.35 \%$ in the SSBE $(100 \mu \mathrm{g} / \mathrm{ml})$-treated PANC-1 cells, suggesting that SSBE significantly increased the number of apoptotic cells in a concentration-dependent manner. In addition, western blot analyses showed that SSBE treatment increased the protein expression of Bax, caspase- 3 and caspase-8, and decreased the protein expression of Bcl-2 (Fig. 1E). Moreover, upregulated gene expression of $\mathrm{Bax}$ and $\mathrm{Bad}$, and downregulated gene expression of $\mathrm{Bcl}-2$ were observed in the PANC-1 cells treated with SSBE (Fig. 1F). Thus, these data indicated that SSBE-induced apoptosis of PCCs may be through a mitochondrial-mediated pathway. This induction of apoptosis of PCCs after SSBE treatment was also identified by the downregulation of mRNA expression of survivin (also called baculoviral inhibitor of apoptosis repeat-containing 5), a member of the inhibitor of apoptosis (IAP) family (Fig. 1F) (19).

Effect of SSBE on the expression of c-Myc and 553 in PCCs. In SSBE-treated PANC-1 cells, the protein expression of c-Myc, determined by western blotting was significantly decreased and the expression of p53 was increased (Fig. 2A). Reduction in c-Myc expression was also confirmed by immunofluorescence staining (Fig. 2B). In addition, downregulated mRNA 
A
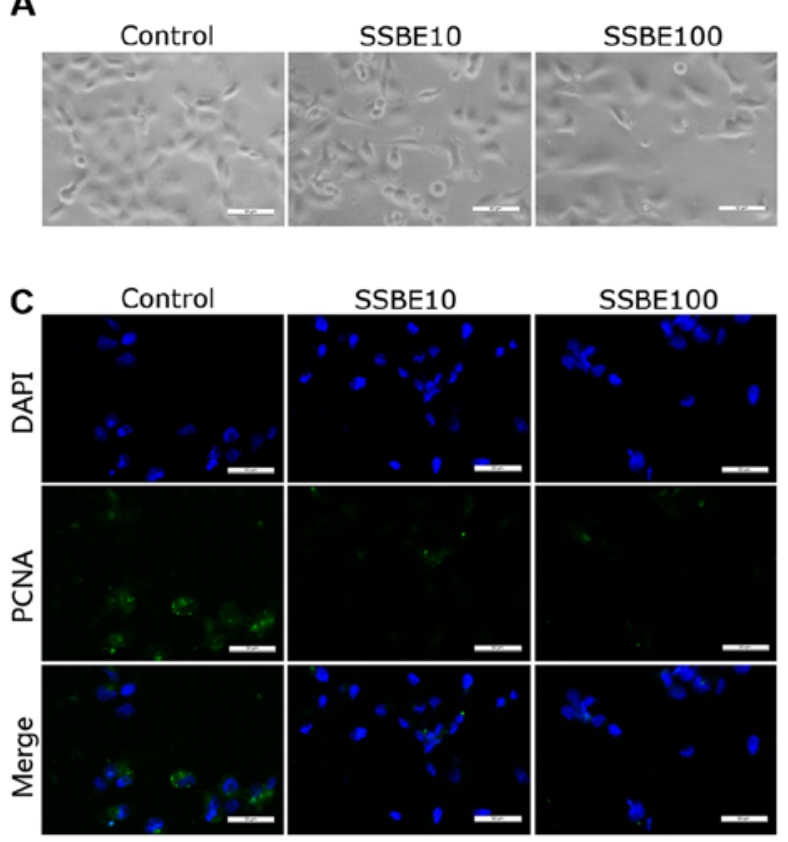

E
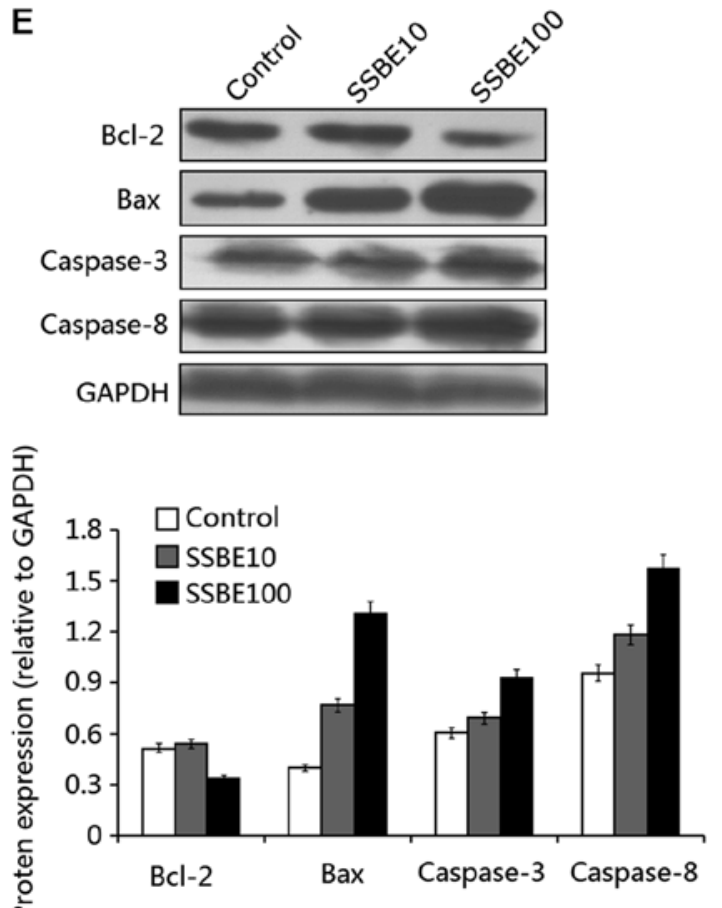

B

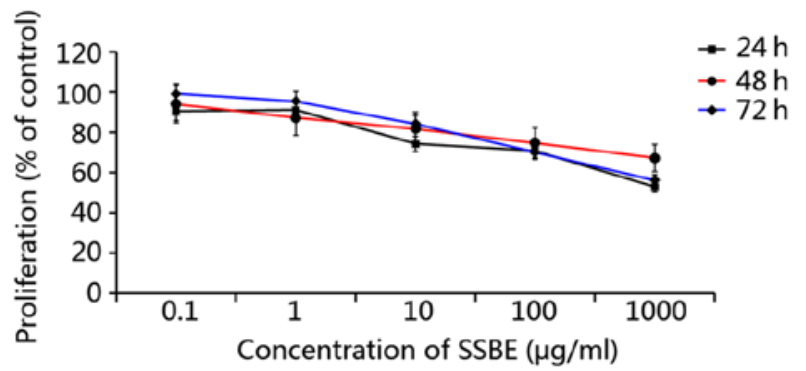

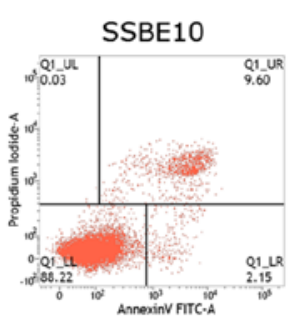
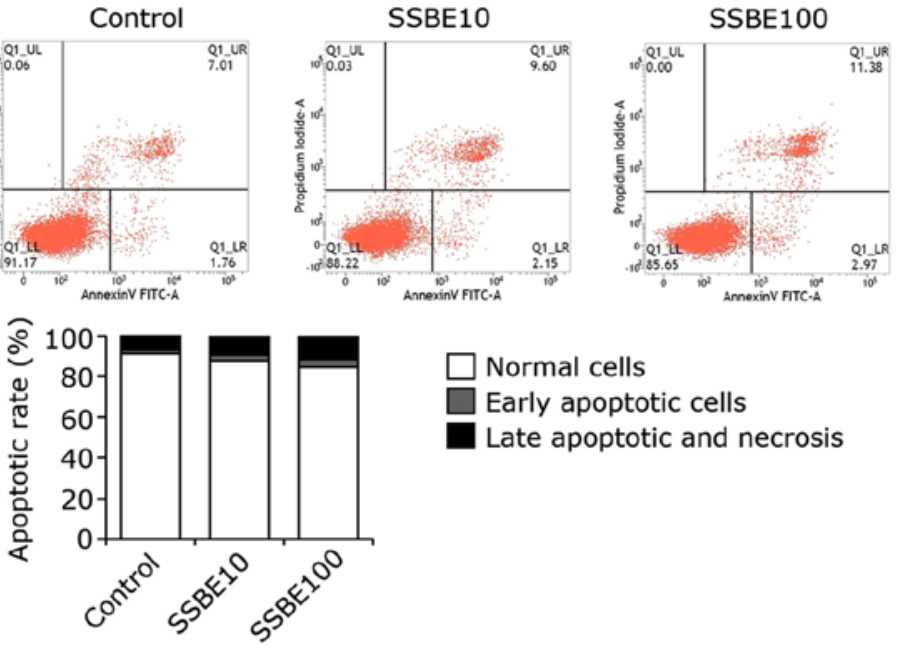

$\mathbf{F}$

造

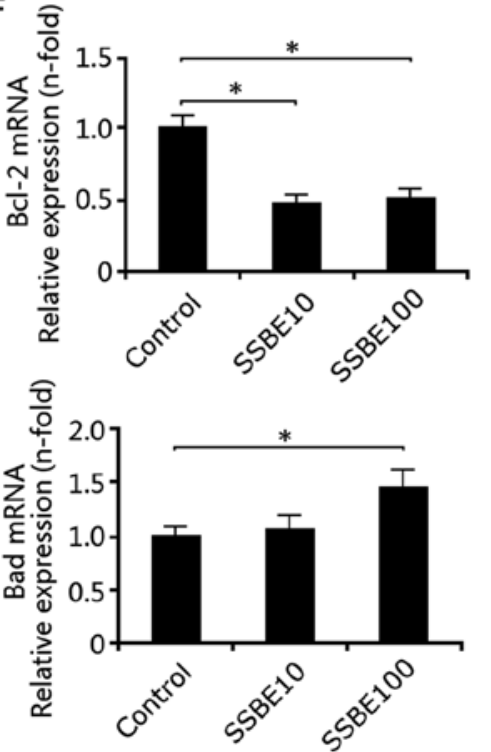

$\square$ Normal cells

$\square$ Early apoptotic cells

Late apoptotic and necrosis
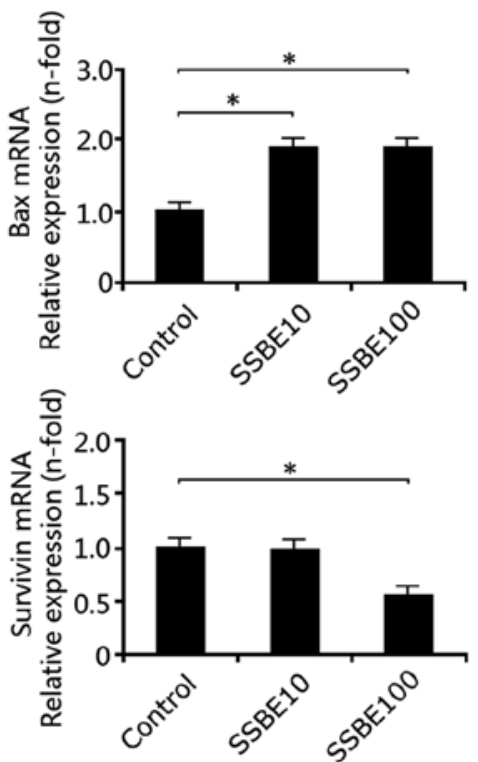

Figure 1. Effect of SSBE on cell proliferation and apoptosis in PCCs. (A) Morphological changes of PCCs with or without SSBE treatment. Scale bar, $50 \mu \mathrm{m}$. (B) CCK-8 assay of the cell proliferation of PCCs with or without treatment of SSBE at different concentrations. (C) Immunofluorescence staining for PCNA in the control and SSBE-treated PCCs. Scale bar, $50 \mu \mathrm{m}$. (D) Flow cytometric analysis for cellular apoptosis in PCCs with or without SSBE treatment. (E) Western blot analyses of Bcl-2, Bax, caspase-3 and caspase-8 in the control and SSBE-treated PCCs. (F) Quantitative RT-PCR showing changes in the gene expression of Bcl-2, Bax, Bad and survivin in PCCs with or without SSBE treatment. Data are represented as the mean \pm SEM ( $n=6)$; ${ }^{*} \mathrm{P}<0.05$ vs. the control group.

expression of Myc and upregulated expression of TP53 were also observed in the PANC-1 cells following SSBE treatment (Fig. 2C). These findings indicated that SSBE inhibits c-Myc expression and promotes p53 expression in PCCs. Previous studies have shown that c-Myc (encoded by the gene Myc) has an important role in apoptosis, cell cycle progression and cellular transformation (20). Enhanced expression of c-Myc induces the unregulated expression of many genes, some of which are involved in cell proliferation. Similarly, as a tumor-suppressor protein, p53 (encoded by the gene Tp53) plays a crucial role in cellular apoptosis, genomic stability and inhibition of angiogenesis (21). Low expression of p53 induces $\mathrm{G} 2 / \mathrm{M}$ transition in various cancer cells. In the present study, SSBE inhibited gene and protein expression of c-Myc and promoted the expression of p53, thereby inducing the cellular apoptosis of PCCs and inhibiting proliferation. 
A
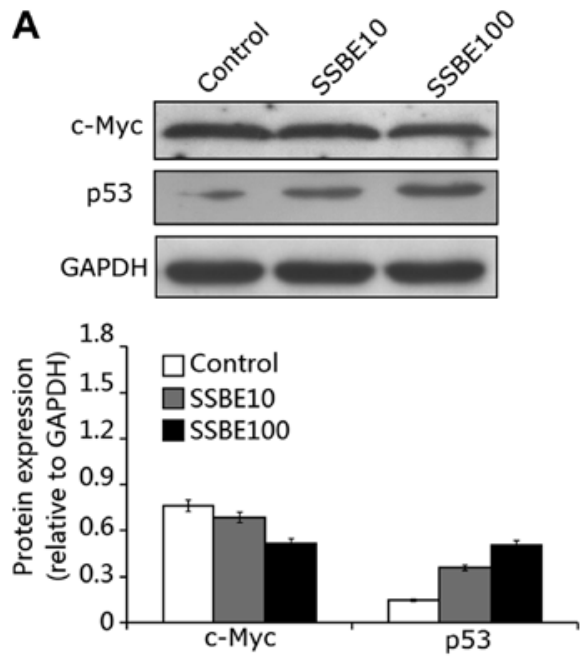

C
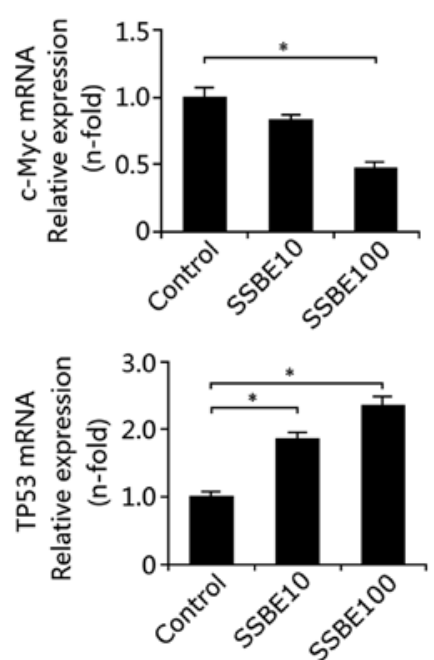

B

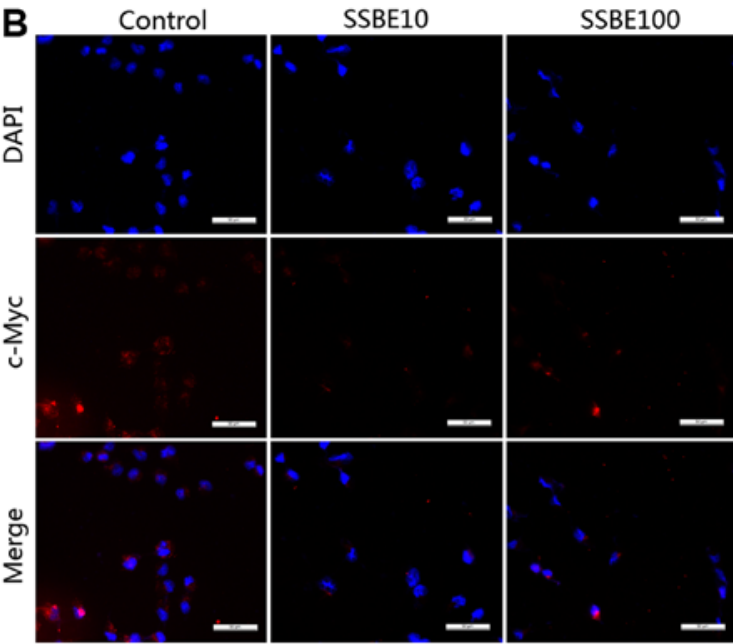

D

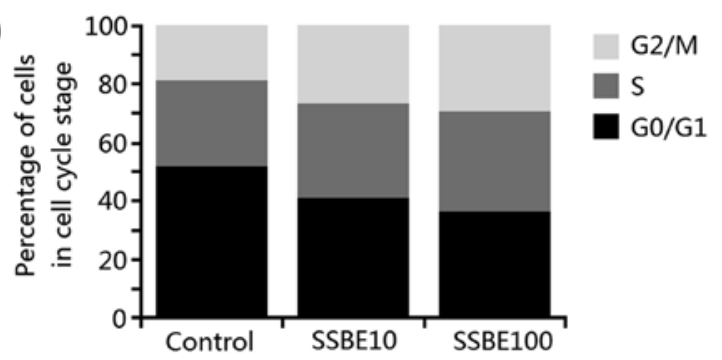

E

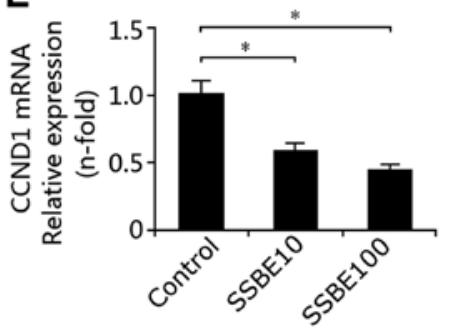

F

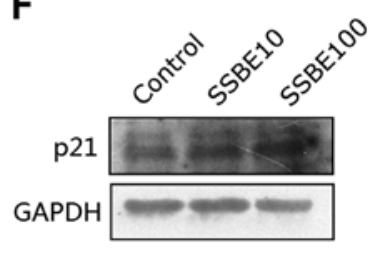

Figure 2. Effect of SSBE on c-Myc and p53 expression and cell cycle distribution in the PCCs. (A) Western blot analyses for c-Myc and p53 in the control and SSBE-treated PCCs. (B) Immunofluorescence staining for c-Myc in the control and SSBE-treated PCCs. Scale bar, $50 \mu \mathrm{m}$. (C) Quantitative RT-PCR showing the changes in gene expression of c-Myc and TP53 in the PCCs with or without SSBE treatment. Data are represented as the mean \pm SEM ( $n=6$ ); ${ }^{*} \mathrm{P}<0.05$ vs. the control group. (D) Flow cytometric analysis of the cell cycle in the control and SSBE-treated PCCs. (E) Quantitative RT-PCR showing the changes in gene expression of CCND1 in PCCs with or without SSBE treatment. Data are represented as the mean \pm SEM $(n=6)$; ${ }^{*} \mathrm{P}<0.05$ vs. the control group. (F) Western blot analyses of 21 in the PCCs with or without SSBE treatment.

Effect of SSBE on cell cycle phase distribution in PCCs. The induction of cellular apoptosis of PCCs and inhibition of proliferation may be a result of cell cycle arrest. Thus, we examined the effect of SSBE on the cell cycle. Flow cytometric analysis showed that the percentage of G0/G1 phase PCCs in the SSBE-treated PCCs was significantly decreased compared with this percentage in the controls, and the percentage of G2/M-phase cells was increased (Fig. 2D), indicating that SSBE treatment induced excessive accumulation and cell cycle arrest of PCCs at the G2/M phase. Further study showed that the SSBE-induced cell cycle arrest of PCCs was through the downregulation of the expression of cyclin D1 (encoded by the gene CCND1) (Fig. 2E) and upregulation of the expression of cell cycle inhibitor p21 (Fig. 2F). Cyclin D1 is a protein required for progression through the G1 phase of the cell cycle (22). As a regulatory subunit of cyclin-dependent kinases CDK4, the cyclin D1-CDK4 complex promotes passage through the
G1 phase by inhibiting the retinoblastoma protein $(\mathrm{pRb})$, and enables the activation of cyclin E-CDK 2 complex by sequestering CDK inhibitory protein p21 (23). SSBE-induced changes in gene and protein expression of PCCs drive cell cycle arrest at the $\mathrm{G} 2 / \mathrm{M}$ phase.

Effect of SSBE on EMT in PCCs. EMT is a process whereby epithelial cells lose their epithelial phenotypes such as E-cadherin, and regain new characteristic features of mesenchymal molecules including $\alpha$-smooth muscle actin $(\alpha$-SMA) (24). EMT is critical for tumor invasion and metastasis (25). In the present study, the gene and protein expression levels of $\alpha$-SMA in PANC-1 cells were downregulated by SSBE treatment (Fig. 3A and B). Additionally, the expression of E-cadherin was upregulated in the SSBE-treated cells (Fig. 3B). Thus, SSBE inhibited the EMT process of PCCs, resulting in the reduction in the ability for invasion and metastasis. 

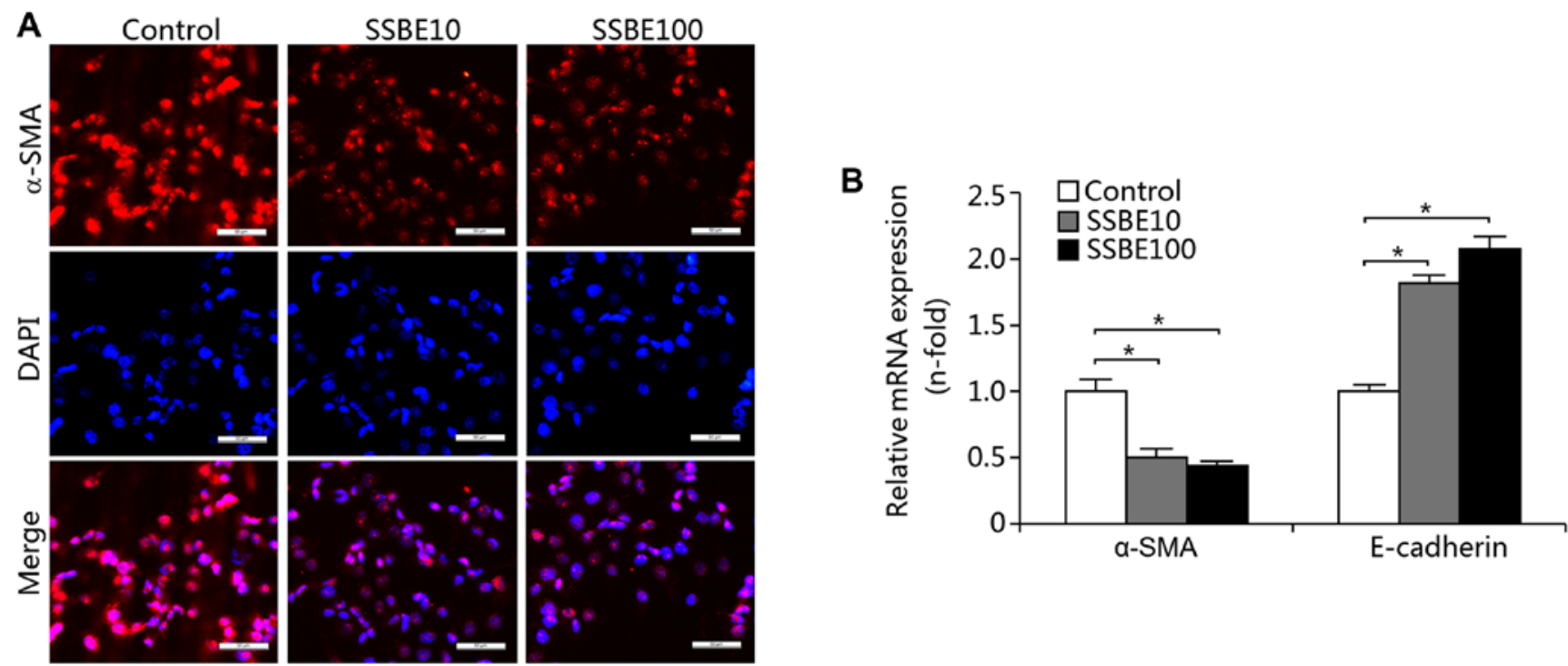

Figure 3. Effect of SSBE on EMT in PCCs. (A) The expression of $\alpha$-SMA was determined by immunofluorescence staining. Scale bar, $50 \mu \mathrm{m}$. (B) Quantitative RT-PCR showing the changes in gene expression of $\alpha$-SMA and E-cadherin in PCCs with or without SSBE treatment. Data are represented as the mean \pm SEM $(\mathrm{n}=6) ;{ }^{*} \mathrm{P}<0.05$ vs. the control group.
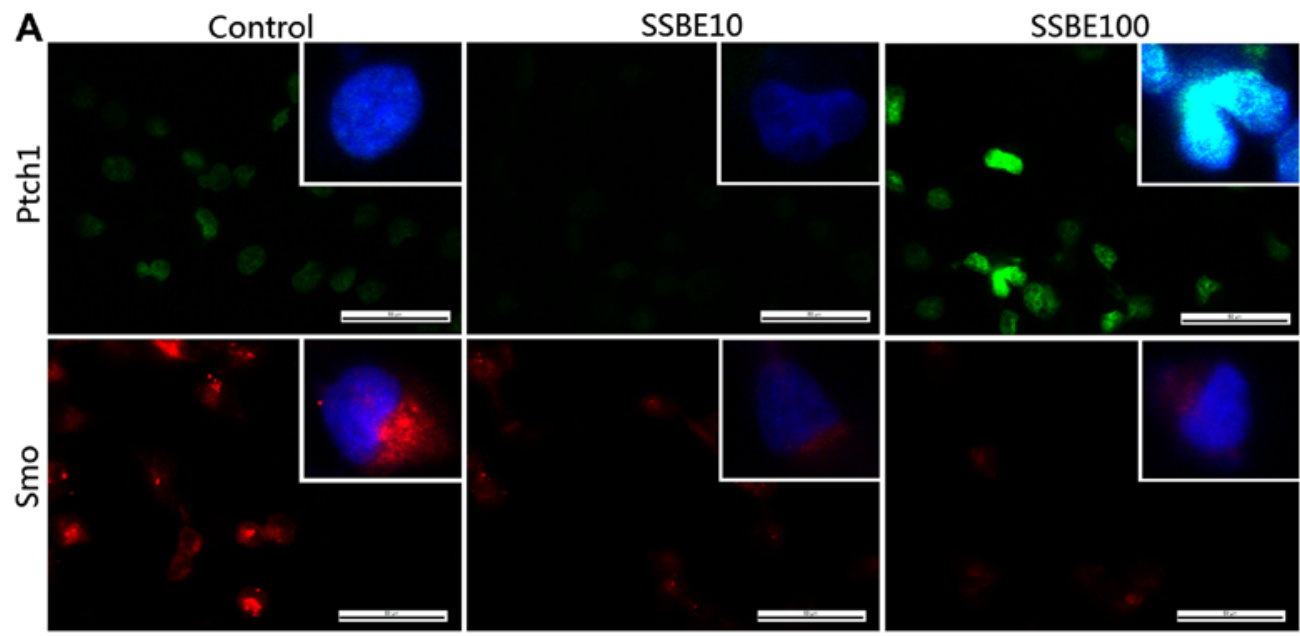

B

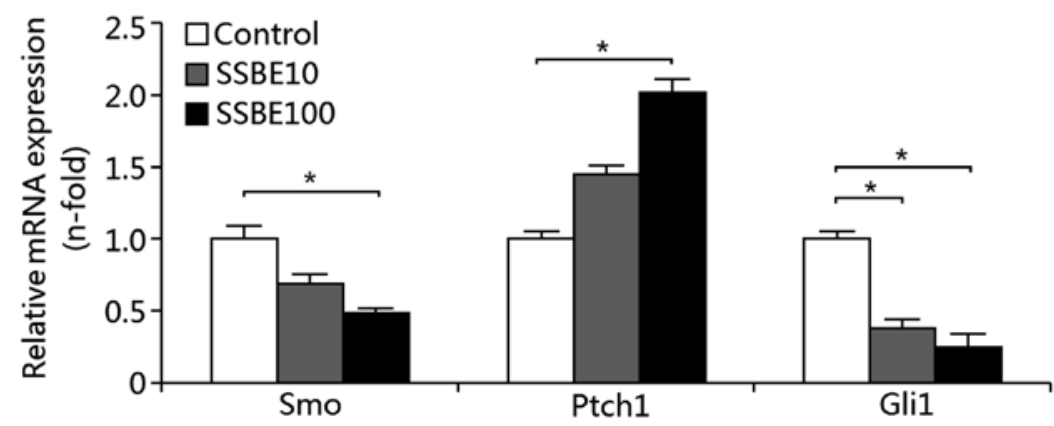

Figure 4. Effect of SSBE on Hedgehog signaling in PCCs. (A) The expression of Ptch1 and Smo was determined by immunofluorescence staining. Scale bar, $50 \mu \mathrm{m}$. (B) Quantitative RT-PCR showing the changes in gene expression of Ptch1, Smo and Gli1 in PCCs with or without SSBE treatment. Data are represented as the mean $\pm \operatorname{SEM}(\mathrm{n}=6) ;{ }^{*} \mathrm{P}<0.05$ vs. the control group.

Effect of SSBE on the activity of Hedgehog signaling in PCCs. As mentioned above, SSBE has an inhibitory effect on the proliferation of PCCs. This inhibition of proliferation may be associated with the activation of proliferation-related signaling.
Thus, we examined the activity of the Hedgehog signaling pathway in the SSBE-treated PANC-1 cells. Our results showed that SSBE treatment increased the protein expression of Ptch1 as determined by immunofluorescence staining (Fig. 4A) and 


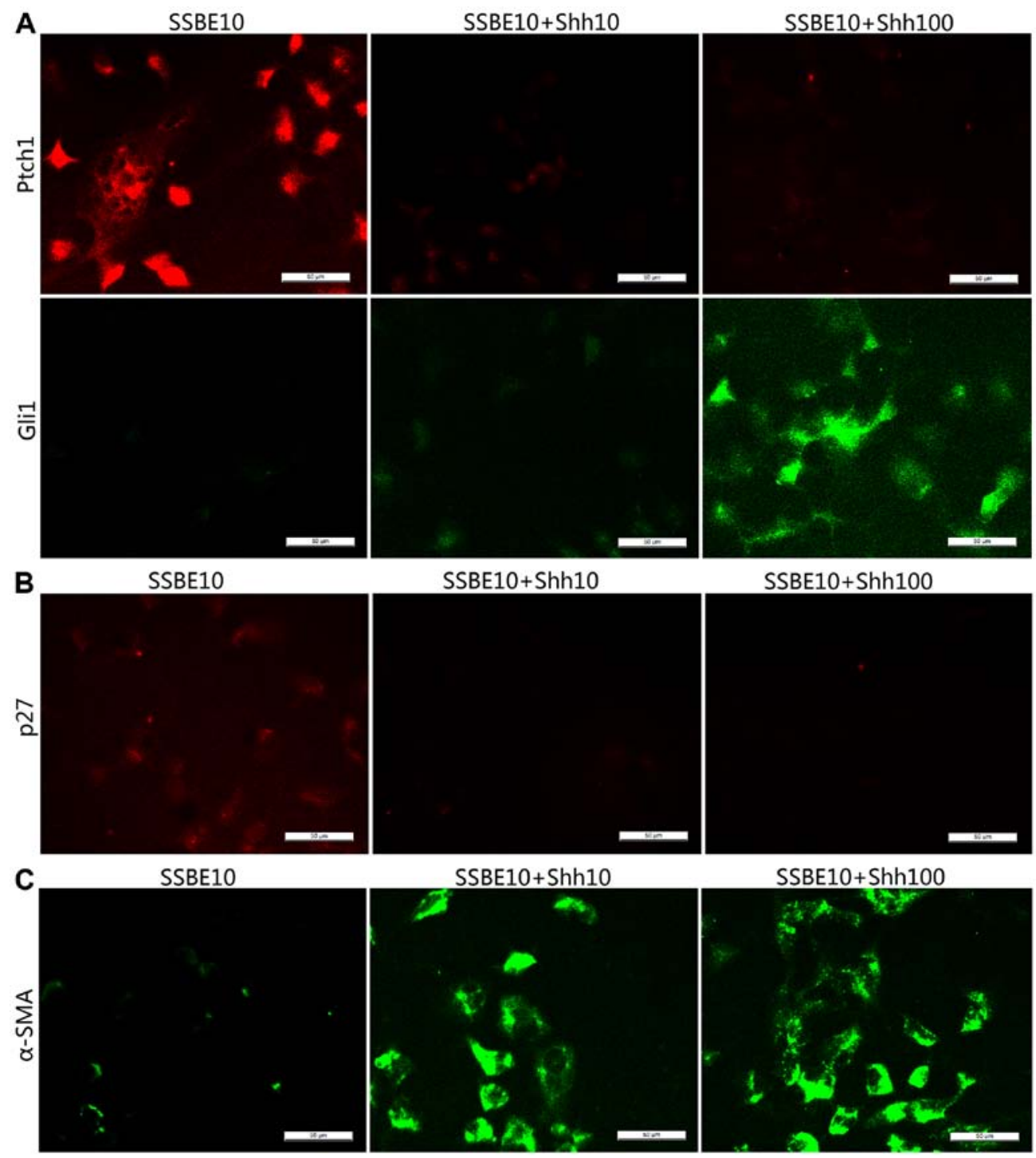

Figure 5. Effect of activated Hedgehog signaling on SSBE-treated PCCs. (A) The expression of Ptch1 and Gli1 was determined by immunofluorescence staining. Scale bar, $50 \mu \mathrm{m}$. (B) The expression of $\mathrm{p} 27$ was determined by immunofluorescence staining. Scale bar, $50 \mu \mathrm{m}$. (C) The expression of $\alpha$-SMA was determined by immunofluorescence staining. Scale bar, $50 \mu \mathrm{m}$.

the gene expression as determined by qRT-PCR (Fig. 4B). As a crucial receptor of canonical Hedgehog signaling, enhanced Ptch1 expression inhibits the activation of signaling. In addition to these, SSBE also decreased the expression of Smo and Gli1 (Fig. 4A and B), which are targets of activated Hedgehog signaling, suggesting that activity of the Hedgehog pathway in PCCs was reduced by SSBE treatment. As a result, SSBE inhibits the proliferation of PCCs.

Effect of activated Hedgehog signaling on SSBE-mediated inhibition of PCC proliferation. Since SSBE treatment exerts its inhibitory effect on the activation of the Hedgehog signaling pathway in PCCs, we ascertained whether regulation of Hedgehog signaling is involved in SSBE-mediated inhibition. In the present study, exogenous recombinant protein sonic Hedgehog (Shh) was used to activate Hedgehog signaling in the SSBE-treated PANC-1 cells. We found that Shh abolished SSBE-mediated upregulated expression of Ptch1 and downregulated expression of Gli1 (Fig. 5A), indi- cating that Hedgehog signaling was reactivated, resulting in the reduction of p27 and $\alpha$-SMA (Fig. 5B and C). p27, also called Kip1, regulates the cell cycle by inhibiting the checkpoint kinase cdk2/cyclin E and blocking cell cycle progression through G1/S transition. Downregulated expression of p27 abolishes cell cycle arrest, and thereby promotes proliferation and inhibits apoptosis. Thus, our in vitro experiment indicated that the Hedgehog signaling pathway plays an important role in SSBE-mediated inhibition of PCC proliferation and EMT.

Effect of SSBE on Hedgehog signaling activity in animal xenograft models of pancreatic cancer. To assess whether similar anticancer effects also occur in vivo, SSBE (10 and $100 \mathrm{mg} /$ $\mathrm{kg} \cdot$ day) was administered continuously for 30 days to mice subjected to injection of PCCs. Fig. 6A shows the morphological changes in the experimental groups as a result of SSBE treatment. H\&E staining identified the pathological results of pancreatic cancer in tissues of the model group (Fig. 6B) 
A

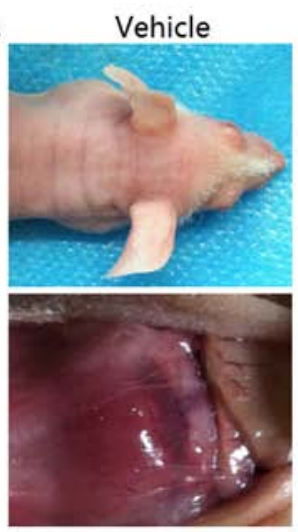

B
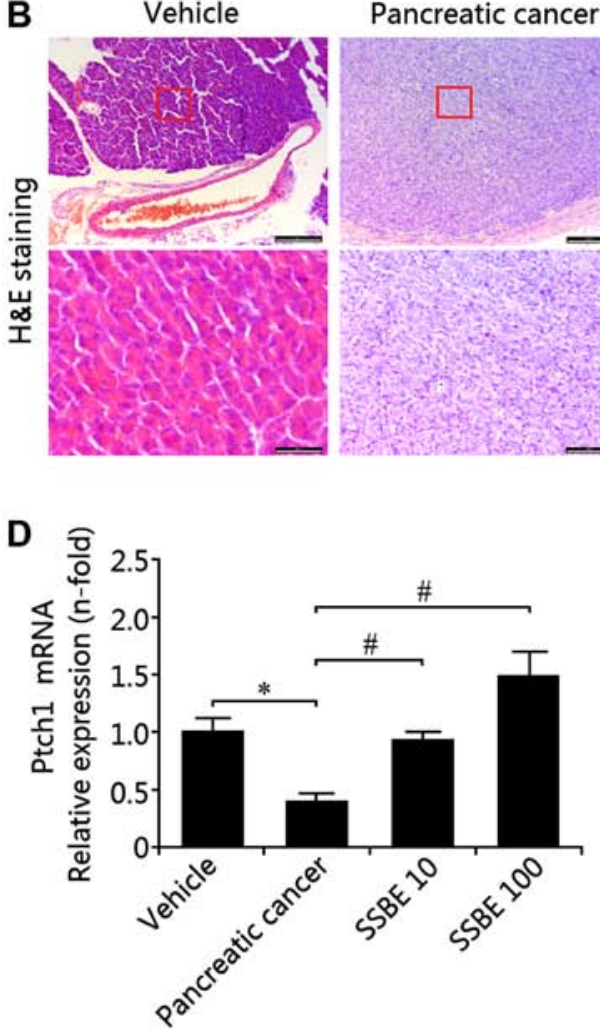

Pancreatic cancer

Pancreatic cancer
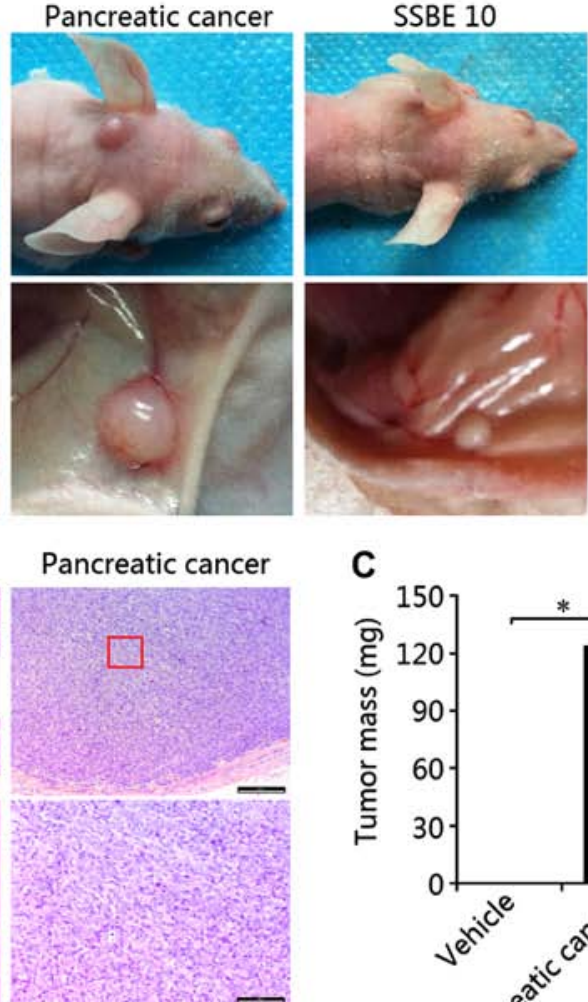

C
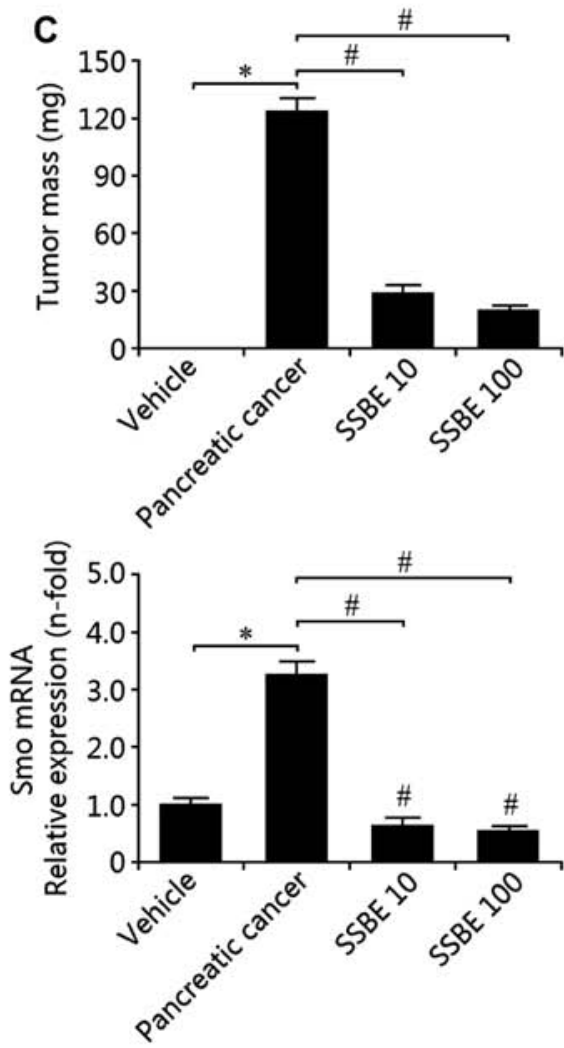

Figure 6. Effect of SSBE on Hedgehog signaling in animal xenograft models of pancreatic cancer. (A) Morphological changes in the SSBE-treated groups compared with the vehicle groups. (B) Pathological results of pancreatic tissues in the different groups were assessed by H\&E staining. Scale bar, $200 \mu \mathrm{m}$ (upper panels); scale bar, $50 \mu \mathrm{m}$ (lower panels). (C) Tumor mass of pancreatic tissues in the different groups. (D) Quantitative RT-PCR showing the changes in gene expression of Ptch1 and Smo in the different groups. Data in the present study are represented as the mean \pm SEM ( $=6$ ), ${ }^{*} \mathrm{P}<0.05$ vs. the vehicle group; ${ }^{\#} \mathrm{P}<0.05$ vs. the pancreatic cancer group.

and the tissue mass of the model group was significantly increased when compared with the vehicle group (Fig. 6C). Following SSBE treatment, the mass of the tumor tissues was significantly decreased in a concentration-dependent manner (Fig. 6C). Thus, these findings suggest that SSBE exerts its inhibitory effect on tumor growth. Further study showed that the mRNA expression of Ptch1 was decreased in the model group, and the expression of Smo was increased, indicating that Hedgehog signaling was activated in the occurrence and development of pancreatic cancer (Fig. 6D). This activation of Hedgehog signaling in the model group was inhibited by SSBE administration. Therefore, these findings reconfirmed that SSBE-mediated antitumor activity may be through suppression of Hedgehog signaling.

\section{Discussion}

In the present study, we investigated the effects of SSBE on pancreatic cancer using a cell culture system and an experimental mouse model. In cultured PCCs, SSBE exerted its protective effects by inhibiting cell proliferation and inducing marked apoptosis. This SSBE-mediated apoptosis appeared to be through a mitochondrial-dependent pathway. In addition, SSBE treatment induced cell cycle arrest at the G2/M phase by upregulating the expression of $\mathrm{p} 21$. Our further study showed that cell proliferation of PCCs was triggered by the activation of Hedgehog signaling, which was inhibited by SSBE treatment. In the animal xenograft models of pancreatic cancer, the activity of Hedgehog signaling was increased, but was 
inhibited by SSBE administration. Thus, our results provide a rationale for the use of SSBE as a potential supplemental treatment to attenuate pancreatic cancer.

Hedgehog signaling is a stem-related pathway that plays a key role during embryogenesis and tissue regeneration (26). Aberrant activation of the Hedgehog signaling pathway results in pathological consequences, including a variety of human tumors, such as pancreatic cancer (27). In pancreatic tissues after injury, activation of the Hedgehog pathway is triggered through binding of the ligands, including Shh, to its membrane receptor patched1 (Ptch1). The binding of ligands to Ptch1 relieves signal transducer Smoothened (Smo) and activates a cascade that leads to translocation of the transcription factor Gli1 to the nucleus (28). As a result, activated Hedgehog signaling promotes cell proliferation and tissue regeneration by upregulating the expression of downstream target genes, such as c-Myc and cyclin D1 (29). Thus, the Hedgehog signaling pathway is important for the occurrence and development of pancreatic cancer.

In patients with pancreatic cancer, the level of plasma Shh was found to be higher than that in healthy individuals (30). Enhanced levels of plasma Shh were also found in various PCCs (31). In addition, abnormal expression of Ptch1 and Smo are identified to be associated with tumor size, tumor differentiation, lymph node metastasis and clinical stage (32). Furthermore, aberrant methylation of the human Hedgehog interacting protein (HHIP) gene contributes to increased Hedgehog signaling and is closely related to pancreatic neoplasms (33). Thus, these findings elucidate the crucial role of the Hedgehog signaling pathway in pancreatic cancer. In the present study, upregulated gene and protein expression of Smo and Gli1, and downregulated expression of Ptch1 in PCCs were observed. Additionally, activated Hedgehog signaling was also confirmed in the animal xenograft models of pancreatic cancer. Further study showed that this activation of Hedgehog signaling may result in abnormal expression of downstream target genes. For instance, enhanced c-Myc expression and reduced p53 expression were observed in pancreatic cancer cells. The target genes subsequently regulated the p21 expression, which controls the cell cycle. As a result, Hedgehog signaling-mediated hyperproliferation of pancreatic cells results in carcinogenesis. These findings reconfirmed the importance of Hedgehog signaling in pancreatic cancer, and blockade of this pathway may be a strategy for the treatment of pancreatic cancer $(34,35)$.

In the present study, we revealed that SSBE, a traditional Chinese herbal medicine, has a marked anti-pancreatic cancer activity. To date, few studies have investigated the anticancer activity of SSBE. A study by Huang et al reported that SSBE has potential for preventing and inhibiting hepatocellular carcinoma growth, which is associated with the apoptosis of cancer cells (36). In addition, the mRNA and protein expressions of vascular endothelial growth factor (VEGF) and the protein level of p-STAT3 were significantly decreased after SSBE treatment. Thus, SSBE may have an anti-angiogenesis and apoptotic induction effect on PCCs. Our findings also confirmed the above conclusion that SSBE inhibited the proliferation and induced marked apoptosis of PCCs. Our further study revealed that this inhibitory effect of SSBE on PCCs may be associated with cell cycle arrest at the G2/M phase by upregulating p21 expression. SSBE-mediated overexpression of p21 was negatively correlated with c-Myc expression, but positively correlated with p53 expression. In addition, SSBE decreased the gene expression of cyclin D1, aggravating the inhibitory effect on the cell cycle. Moreover, SSBE also suppressed the EMT response of PCCs, thereby reducing the ability of invasion and metastasis of tumors. In animal xenograft models of pancreatic cancer, SSBE markedly inhibited tumor growth. Thus, SSBE may be an effective therapeutic drug for pancreatic cancer.

Further study revealed that SSBE exerted its anticancer effects by antagonizing the activation of Hedgehog signaling. Like other studies, our study also identified activated Hedgehog signaling in PCCs and in animal xenograft models of pancreatic cancer. With the treatment of SSBE, the activation of Hedgehog signaling was inhibited in a concentration-dependent manner. In cultured PCCs, exogenous recombinant protein Shh was used to induce the activation of Hedgehog signaling, and resulted in the abolishment of apoptotic induction and EMT response. In pancreatic cancer models, SSBE administration also suppressed the activity of Hedgehog signaling. Thus, Hedgehog signaling may be a crucial mechanism for the SSBE-mediated anticancer effects. Our study also supported the conclusion that inhibition of Hedgehog signaling has a protective effect on pancreatic cancer (34). However, it is necessary to note that the clinical application of SSBE needs more investigation, including safety and effectiveness. In addition, as a Chinese herbal medicine, SSBE contains many complicated and uncertain chemical components, and thereby may exhibit unpredictable and complex effects, thus the drug dosage and molecular mechanisms of SSBE must be elucidated.

In conclusion, SSBE exerts its pancreatic anticancer activity by suppressing the Hedgehog pathway in vitro and in vivo, resulting in the inhibition of proliferation and induction of apoptosis of PCCs. Thus, SSBE has potential for the treatment of pancreatic cancer.

\section{Acknowledgements}

The present study was supported in part by research grants from the Natural Science Foundation of China (30772548), and the Foundation from the Excellent Master Dissertation of the College of Laboratory Medicine at Chongqing Medical University (201202).

\section{References}

1. Douglass HO Jr: Adjuvant therapy for pancreatic cancer. World J Surg 19: 270-274, 1995.

2. Kayahara M, Funaki K, Tajima H, Takamura H, Ninomiya I, Kitagawa $\mathrm{H}$ and Ohta T: Surgical implication of micrometastasis for pancreatic cancer. Pancreas 39: 884-888, 2010.

3. Richter J and Saif MW: Updates in adjuvant therapy in pancreatic cancer: Gemcitabine and beyond. Highlights from the '2010 ASCO Gastrointestinal Cancers Symposium'. Orlando, FL, USA. January 22-24, 2010. JOP 11: 144-147, 2010.

4. Woo JH, Li D, Wilsbach K, Orita H, Coulter J, Tully E, Kwon TK, $\mathrm{Xu}$ S and Gabrielson E: Coix seed extract, a commonly used treatment for cancer in China, inhibits NFkappaB and protein kinase C signaling. Cancer Biol Ther 6: 2005-2011, 2007.

5. Habib SH, Makpol S, Abdul Hamid NA, Das S, Ngah WZ and Yusof YA: Ginger extract (Zingiber officinale) has anti-cancer and anti-inflammatory effects on ethionine-induced hepatoma rats. Clinics 63: 807-813, 2008. 
6. Heo BG, Park YS, Chon SU, Lee SY, Cho JY and Gorinstein S Antioxidant activity and cytotoxicity of methanol extracts from aerial parts of Korean salad plants. Biofactors 30: 79-89, 2007.

7. Jung HJ, Kang HJ, Song YS, Park EH, Kim YM and Lim CJ: Anti-inflammatory, anti-angiogenic and anti-nociceptive activities of Sedum sarmentosum extract. J Ethnopharmacol 116: 138-143, 2008

8. Morikawa T, Zhang Y, Nakamura S, Matsuda H, Muraoka O and Yoshikawa M: Bioactive constituents from Chinese natural medicines. XXII. Absolute structures of new megastigmane glycosides, sedumosides $\mathrm{E}_{1}, \mathrm{E}_{2}, \mathrm{E}_{3}, \mathrm{~F}_{1}, \mathrm{~F}_{2}$, and $\mathrm{G}$, from Sedum sarmentosum (Crassulaceae). Chem Pharm Bull 55: 435-441, 2007.

9. Ninomiya K, Morikawa T, Zhang Y, Nakamura S, Matsuda H, Muraoka $\mathrm{O}$ and Yoshikawa M: Bioactive constituents from Chinese natural medicines. XXIII. Absolute structures of new megastigmane glycosides, sedumosides $\mathrm{A}_{4}, \mathrm{~A}_{5}, \mathrm{~A}_{6}, \mathrm{H}$, and I, and hepatoprotective megastigmanes from Sedum sarmentosum. Chem Pharm Bull 55: 1185-1191, 2007.

10. Oh H, Kang DG, Kwon JW, Kwon TO, Lee SY, Lee DB and Lee HS: Isolation of angiotensin converting enzyme (ACE) inhibitory flavonoids from Sedum sarmentosum. Biol Pharm Bull 27: 2035-2037, 2004.

11. Johari J, Kianmehr A, Mustafa MR, Abubakar S and Zandi K: Antiviral activity of baicalein and quercetin against the Japanese encephalitis virus. Int J Mol Sci 13: 16785-16795, 2012.

12. Wang G, Song L, Wang H and Xing N: Quercetin synergizes with 2-methoxyestradiol inhibiting cell growth and inducing apoptosis in human prostate cancer cells. Oncol Rep 30: 357-363, 2013.

13. Youn H, Jeong JC, Jeong YS, Kim EJ and Um SJ: Quercetin potentiates apoptosis by inhibiting nuclear factor-kappaB signaling in H460 lung cancer cells. Biol Pharm Bull 36: 944-951, 2013.

14. Mahmoud MF, Hassan NA, El Bassossy HM and Fahmy A: Quercetin protects against diabetes-induced exaggerated vasoconstriction in rats: Effect on low grade inflammation. PLoS One 8: e63784, 2013.

15. Sun J, Sun G, Meng X, Wang H, Luo Y, Qin M, Ma B, Wang M, Cai D, Guo P, et al: Isorhamnetin protects against doxorubicininduced cardiotoxicity in vivo and in vitro. PLoS One 8: e64526, 2013.

16. Bai Y, Lu H, Zhang G, Wu C, Lin C, Liang Y and Chen B: Sedum sarmentosum Bunge extract exerts renal anti-fibrotic effects in vivo and in vitro. Life Sci 105: 22-30, 2014.

17. Bai Y, Lu H, Wu C, Liang Y, Wang S, Lin C, Chen B and Xia P. Resveratrol inhibits epithelial-mesenchymal transition and renal fibrosis by antagonizing the hedgehog signaling pathway. Biochem Pharmacol 92: 484-493, 2014.

18. Yu F, Guo Y, Chen B, Dong P and Zheng J: MicroRNA-17-5p activates hepatic stellate cells through targeting of Smad7. Lab Invest 95: 781-789, 2015.

19. Athanasoula KC, Gogas H, Polonifi K, Vaiopoulos AG, Polyzos A and Mantzourani M: Survivin beyond physiology: Orchestration of multistep carcinogenesis and therapeutic potentials. Cancer Lett 347: 175-182, 2014.
20. McMahon SB: MYC and the control of apoptosis. Cold Spring Harb Perspect Med 4: a014407, 2014.

21. Taylor WR and Stark GR: Regulation of the G2/M transition by p53. Oncogene 20: 1803-1815, 2001 .

22. Baldin V, Lukas J, Marcote MJ, Pagano M and Draetta G: Cyclin D1 is a nuclear protein required for cell cycle progression in G1. Genes Dev 7: 812-821, 1993.

23. Diehl JA: Cycling to cancer with cyclin D1. Cancer Biol Ther 1: 226-231, 2002.

24. Thiery JP, Acloque H, Huang RY and Nieto MA: Epithelialmesenchymal transitions in development and disease. Cell 139: 871-890, 2009.

25. Savagner P: The epithelial-mesenchymal transition (EMT) phenomenon. Ann Oncol 21 (Suppl 7): vii89-vii92, 2010.

26. Edwards PC, Ruggiero S, Fantasia J, Burakoff R, Moorji SM, Paric E, Razzano P, Grande DA and Mason JM: Sonic hedgehog gene-enhanced tissue engineering for bone regeneration. Gene Ther 12: 75-86, 2005.

27. Thayer SP, di Magliano MP, Heiser PW, Nielsen CM, Roberts DJ, Lauwers GY, Qi YP, Gysin S, Fernández-del Castillo C, Yajnik V, et al: Hedgehog is an early and late mediator of pancreatic cancer tumorigenesis. Nature 425: 851-856, 2003.

28. Varjosalo M and Taipale J: Hedgehog: Functions and mechanisms. Genes Dev 22: 2454-2472, 2008

29. Katoh $Y$ and Katoh M: Hedgehog signaling pathway and gastric cancer. Cancer Biol Ther 4: 1050-1054, 2005.

30. El-Zaatari M, Daignault S, Tessier A, Kelsey G, Travnikar LA, Cantu EF, Lee J, Plonka CM, Simeone DM, Anderson MA, et al: Plasma Shh levels reduced in pancreatic cancer patients. Pancreas 41: 1019-1028, 2012.

31. Li X, Wang Z, Ma Q, Xu Q, Liu H, Duan W, Lei J, Ma J, Wang X, Lv S, et al: Sonic hedgehog paracrine signaling activates stromal cells to promote perineural invasion in pancreatic cancer. Clin Cancer Res 20: 4326-4338, 2014.

32. Yang Y, Tian X, Xie X, Zhuang Y, Wu W and Wang W: Expression and regulation of hedgehog signaling pathway in pancreatic cancer. Langenbecks Arch Surg 395: 515-525, 2010.

33. Martin ST, Sato N, Dhara S, Chang R, Hustinx SR, Abe T, Maitra A and Goggins M: Aberrant methylation of the human Hedgehog interacting protein (HHIP) gene in pancreatic neoplasms. Cancer Biol Ther 4: 728-733, 2005.

34. Xu Y, An Y, Wang X, Zha W and Li X: Inhibition of the Hedgehog pathway induces autophagy in pancreatic ductal adenocarcinoma cells. Oncol Rep 31: 707-712, 2014.

35. Guo J, Gao J, Li Z, Gong Y, Man X, Jin J and Wu H: Adenovirus vector-mediated Glil siRNA induces growth inhibition and apoptosis in human pancreatic cancer with Smo-dependent or Smo-independent $\mathrm{Hh}$ pathway activation in vitro and in vivo. Cancer Lett 339: 185-194, 2013.

36. Huang D, Zhang W, Huang D and Wu J: Antitumor activity of the aqueous extract from Sedum sarmentosum Bunge in vitro. Cancer Biother Radiopharm 25: 81-88, 2010. 